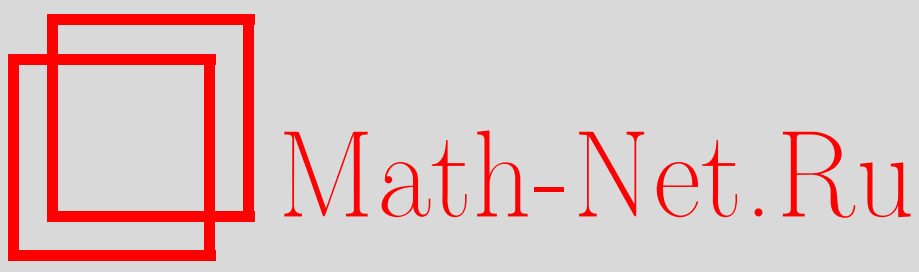

С. И. Богатая, С. А. Богатый, Х. Цишанг, О композициях открытых отображений, Матем. сб., 2002, том 193, номер 3, 3-20

DOI: https://doi.org/10.4213/sm632

Использование Общероссийского математического портала Math-Net.Ru подразумевает, что вы прочитали и согласны с пользовательским соглашением http://www . mathnet.ru/rus/agreement

Параметры загрузки:

IP: 54.166 .219 .16

26 апреля 2023 г., $11: 30: 14$ 
УДК 515.162 .2

\author{
С.И. Богатая, С. А. Богатый, Х. Цишанг
}

\title{
О композициях открытых отображений
}

\begin{abstract}
Построено 4-кратное накрытие поверхности рода 2 поверхностью рода 5 , которое не представляется в виде композиции двух открытых нетривиальных отображений, что показывает неполноту препятствия Баилдона. Доказываются различные теоремы о разложимости регулярного накрытия в композицию (регулярных) накрытий разных кратностей в разных последовательностях. Для разветвленных накрытий предложено новое препятствие к разложимости - массив ветвления точек ветвления.

Библиографйл: 62 названия.
\end{abstract}

\section{Введение}

Борсук и Мольски показали [1], что всякое локально взаимно однозначное отображение компакта (т.е. компактного метрического пространства) является композицией конечного числа простых отображений (т.е. отображений кратности 2). Сиеклуцкий доказал [2], что всякое отображение конечной кратности конечномерного компакта является композиичей простых отображсений. Кржемпек улучшил [3] теорему Сиеклуцкого: всякое замкнутое отображсние кратности $\leqslant k$, заданное на $n$-мерном метрическом пространстве, является композичией не более чем $(n+1) k-1$ простых замкнутых отображсений метризуемых пространств.

Ситуация с открытыми отображениями совершенно иная. Кржемпек доказал [3], что всякое открытое (открыто-замкнутое) сюрвективное отображсение $f: X \rightarrow Y$ кратности $\leqslant k+1$, заданное на нульмерном метрическом пространстве $X$, является композицией $f_{k} \circ \cdots \circ f_{1} k$ простых открытых (открыто-замкнутых) сюрвективных отображсений $f_{1}, \ldots, f_{k}$. Дыдак изучил [4] 1-мерный случай: отображение $z \mapsto z^{p}$ единичной окружности на комплексной плоскости не является композицией локально взаимно однозначных отображсений порядк $a \leqslant p-1$. Баилдон получил [5] препятствие к разложимости отображения в композицию открытых отображений в 2 -мерном случае. Пусть $S_{g}$ означает замкнутую ориентируемую поверхность рода $g$. Баилдон доказал [5; теорема 2], что если открытое отображение $f: S_{h} \rightarrow S_{g}$ является композицией $f_{k} \circ \cdots \circ f_{1} k$ открытых простых сюрвективных отобрахсений, то Ord $f=2^{k}$.

\footnotetext{
Частично работа выполнена в ноябре-декабре 2000 г. во время визита второго автора в Ruhr-Universität Bochum в рамках DFG-проекта "Niedrigdimensionale Topologie und geometrisch-topologische Methoden in der Gruppentheorie"; первые два автора поддержаны Российским фондом фундаментальных исследований (грант № 00-01-00289).
}

(C) С. И. БОГАТАЯ, С. А. БОГАТЫЙ, Х. ЦИШАНГ 2002 
Мы переносим теорему Баилдона на композицию произвольных открытых отображений (теорема 2.3) и показываем, что (в отличие от одномерного случая) даже для накрытий поверхностей это арифметическое препятствие на число Ord $f$ не является полньм (следствия 3.3 и $3.8^{\prime}$ ). Доказьваются различные теоремы о разложимости регулярного накрытия в композицию (регулярных) накрытий разных кратностей в разных последовательностях. Даются примеры, показывающие существенность возникаюших ограничений. Для открытых отображений поверхностей с различными кратностями некоторых точек (т.е. разветвленных накрытий) мы предлагаем новое препятствие к разложимости: массив ветвления точек ветвления.

\section{§1. Одномерный случай}

Когда говорится о композиции, первьм назьвается то отображение, которое стоит ближе к аргументу.

ОПРЕДЕЛЕНИЕ 1.1. Говорят, что отображение $f: X \rightarrow Y$ имеет кратность $($ порядок $) \leqslant n \quad(\operatorname{Ord} f \leqslant n)$, если $\left|f^{-1}(y)\right| \leqslant n$ для всякой точки $y \in Y$.

ПрЕДЛОЖЕНИЕ $1.2[6 ; \mathrm{X},(1.2)]$. Если $f: S^{1} \rightarrow S^{1}$ - открытое отображсение, то $n=\operatorname{Ord} f$ конечно $и f$ является $n$-кратным накрытием и топологически сопряхсено отобрахсению $z \mapsto z^{n}$.

ПрЕДЛОЖЕНИЕ 1.3 [6; X, (1.2) и (1.3)]. Если $S^{1} \rightarrow X \rightarrow S^{1}$ - oткрьлтые отображения, то $X=S^{1}$.

Результат Дыдака может быть сформулирован в следующей более сильной форме.

ПРЕДЛОЖЕНИЕ 1.4. Для открытого отображсения $f: S^{1} \rightarrow S^{1}$ следующие условия әквивалентны:

(1) Ord $f=n_{1} \cdots n_{k}$;

(2) $f=f_{k} \circ \cdots \circ f_{1}$, где $S^{1} \stackrel{f_{1}}{\longrightarrow} X_{1} \stackrel{f_{2}}{\longrightarrow} \cdots \stackrel{f_{k-1}}{\longrightarrow} X_{k-1} \stackrel{f_{k}}{\longrightarrow} S^{1}$ являются открытылми отображсениями $u$ Ord $f_{i}=n_{i}$.

ДокАЗАТЕЛЬСтво. $(1) \Rightarrow(2)$. Согласно предложению 1.2 отображение $f$ сопряжено отображению $z^{n}$, которое является композицией отображений $z^{n_{1}}, \ldots, z^{n_{k}}$.

$(2) \Rightarrow(1)$. Согласно предложению 1.3 все компакты $X_{i}$ гомеоморфны окружности, и согласно предложению $1.2 f_{i}$ является накрытием порядка $n_{i}$. Тогда композиция является накрытием порядка $n_{1} \cdots n_{k}$.

Для открытых отображений разветвленных одномерных многообразий (графов) кратность композиции может отличаться от произведения кратностей отображений разложения. Но и в этом случае справедливы некоторые аналоги предложений 1.2 и 1.3 [6; VIII, (7.31)], поэтому справедлив “асимптотический” аналог предложения 1.4 .

\section{§2. Двумерный случай}

Мультипликативность кратности - это “многообразное” свойство. Мы ограничимся рассмотрением ориентируемых поверхностей. 
ПРЕДЛОЖЕНИЕ 2.1 [6; Х, (6.3)]. Если $f: S_{h} \rightarrow S_{g}$ - открытое нульмерное отобрахсение, то $n=\operatorname{Ord} f$ конечно и $f$ является $n$-кратным разветвленным накрытием.

ПРЕДЛОЖЕНИЕ 2.2. Если $S_{h} \rightarrow X \rightarrow S_{g}$ - открытые нульмерные отображения, то $X=S_{q}$ для некоторого $q$.

ДокАЗАТЕльство. Согласно предложению 2.1 отображение $S_{h} \rightarrow X$ является конечнократным и открытым, поэтому $X$ - компактное 2-мерное многообразие (с граничными окружностями или без них) $[6 ; \mathrm{X},(4.2)]$. Кроме того, так как $S_{g}-$ замкнутая ориентируемая поверхность, то $X$ также является замкнутой ориентируемой поверхностью [6; Х, 7, следствие].

ТЕОРема 2.3. Если дана последовательность открытых нульмерных отображсений $S_{h} \stackrel{f_{1}}{\longrightarrow} X_{1} \stackrel{f_{2}}{\longrightarrow} \cdots \stackrel{f_{k-1}}{\longrightarrow} X_{k-1} \stackrel{f_{k}}{\longrightarrow} S_{g}$, то $X_{i}=S_{h_{i}}$ для некоторого $h_{i}$ $u$

$$
\operatorname{Ord}\left(f_{k} \circ \cdots \circ f_{1}\right)=\operatorname{Ord} f_{1} \cdots \operatorname{Ord} f_{k}
$$

ДокАЗАТЕльство. Согласно предложению 2.2 все промежуточные пространства $X_{i}$ являются замкнутыми ориентируемыми поверхностями. Согласно предложению 2.1 все промежуточные отображения являются разветвленными накрытиями и, в частности, имеют максимальную кратность Ord $f_{i}$ во всех точках, кроме конечного множества. Отсюда вытекает мультипликативная формула (M).

В одномерном случае (для многообразий) разложимость числа Ord $f$ является необходимым и достаточным условием разложимости (в классе открытых отображений) отображения $f$. В двумерном случае это не так.

Мы начнем изучение открытых отображений кратности $\leqslant n$ с изучения открытых точно $n$-кратных отображений, т.е. накрытий. Хаймес и Штёккер доказали [7], [8; 3.4$]$ (мы формулируем замкнутую ориентируемую часть их результата), что поверхность $S_{h}$ n-листно накрывает поверхность $S_{g}$ тогда и только тогда, когда $h-1=n(g-1)$. Эта теорема показьвает, что в случае составного числа $n$ сушествует $n$-кратное накрытие $S_{h}$ на $S_{g}$, которое разложимо. Но она оставляет открытым вопрос о существовании такого неразложимого накрытия.

Назовем накрытие $f$ nростылм, если оно не разлагается в композицию двух нетривиальных накрытий. Ясно, что всякое накрытие простого порядка является простым. Индукцией по числу Ord $f$ легко показать, что всякое накрытие разлагается в композицию простых накрытий. Такое разложение ни в коей мере не является единственным: два разложения на простые накрытия могут отличаться даже количеством простых составляющих (разложения (2) и (1) или (3) из теоремы 3.2).

Накрытия над связным и локально линейно связньм полулокально односвязньм пространством $Y$ соответствуют подгруппам фундаментальной групшы $\pi_{1}(Y)$ $[9 ;$ гл. $2, \S 5,13]$. Впредь все пространства предполагаются именно такими, хотя имеется много работ, посвяшенных накрытиям обших пространств [10], [11], которые позволяют формулировать получаемые утверждения в более общем виде. Из мультипликативной формулы (М) следует, что если накрытие поверхностей разложено в композицию открытых отображений, то каждое отображение разложения является накрытием. Вопрос о разложимости общего накрытия сводится к вопросу о всех разложениях некоторого регулярного накрытия (теорема 4.1). Поэтому 
мы будем, в основном, рассматривать задачу разложения регулярных накрытий. Здесь возникают два неэквивалентных случая: накрытия разложения регулярны; некоторые накрытия разложения могут быть нерегулярны. Напомним, что накрытие $p: X \rightarrow Y$ называется регулярным, если для всякого замкнутого пути в $Y$ все его поднятия одновременно замкнуты или одновременно незамкнуты. Известно, что накрытие $p: X \rightarrow Y$ является регулярным тогда и только тогда, когда образ фундаментальной групшы $X$ в фундаментальной группе $Y$ является нормальным делителем, и является регулярным тогда и только тогда, когда на $X$ можно задать такое свободное действие некоторой групшы $G$, что $p$ совпадает с орбитной проекцией. Вопрос разложимости заданного регулярного накрытия $f: X \rightarrow Y$ нам будет удобнее изучать и формулировать не в терминах свойств группы $\pi_{1}(Y)$ и ее нормальной подгруппы $f_{\#}\left(\pi_{1}(X)\right)$, а в терминах группы $G=\pi_{1}(Y) / f_{\#}\left(\pi_{1}(X)\right)$. Последняя группа является в точности группой накрывающих преобразований накрытия $f[9 ;$ гл. $2, \S 6,3]$.

Яворовский доказал [12], что для не более чем двукратного отобрахсения $f: X \rightarrow Y$ следующие условия әквивалентны:

(1) $f$ откритто;

(2) $f$ порождено действием $\mathbb{Z}_{2}$, т.е. $f$ является проекцией на пространство орбит некоторого действия группь $\mathbb{Z}_{2}$ на $X$.

Отсюда вытекает

СлЕДСТВИЕ 2.4. Всякое двукратное накрытие регулярно.

ЗАмЕЧАНИЕ 2.5. Сформулированное следствие является геометрическим эквивалентом следующего алгебраического утверждения: всякая подгруппа индекса 2 является нормальным делителем.

Для заданного действия конечной группы $G$ на компакте $X$ орбитная проекция $p_{G}: X \rightarrow X / G$ обладает (например, согласно теореме Сиеклуцкого-Кржемпека) различными разложениями. Но есть некоторые естественные разложения. Именно всякая подгруппа $H \subset G$ порождает разложение $X \rightarrow Z=X / H \rightarrow Y=X / G$. При этом если подгруппа $H$ является нормальным делителем, то на промежуточном пространстве $Z$ имеется естественное действие факторгруппы $G / H$ и второе отображение разложения также является орбитной проекцией $p_{G / H}: Z \rightarrow$ $Z /(G / H)=X / G$. Оказывается, что для открытых разложений свободных действий это есть общая ситуация.

ТеОрема 2.6. Если орбитная проекиия $p_{G}: X \rightarrow Y=X / G$ свободного действия группы $G$ на пространстве $X$ представлена в виде композичии двух накрытий $f: X \rightarrow Z$ и $g: Z \rightarrow Y$, то первое накрытие регулярно, более того, существует такая подгруппа $H \subset G$, что $f=p_{H}$. При этом накрытие $g$ регулярно тогда и только тогда, когда подгруппа $Н$ является нормальным делителем в $G$.

ДоКАЗАТЕЛЬСтво. Возьмем замкнутый путь $\omega$ в $Z$, и пусть $\omega_{1}$ и $\omega_{2}-$ Два его поднятия в $X$. Тогда $\omega_{1}$ и $\omega_{2}-$ два поднятия в $X$ замкнутого пути $g \circ \omega$ в $Y$. Следовательно, оба эти поднятия $\omega_{1}$ и $\omega_{2}$ одновременно замкнуты или незамкнуты. Но это и означает, что накрытие $f$ регулярно. Регулярность $f$ означает, что на $X$ можно задать такое свободное действие некоторой групшы $H$, что $f=p_{H}$. Группа $H$ является группой накрывающих преобразований накрытия $f$, следовательно, 
и накрытия $p_{G}$. Последнее и означает, что группа $H$ естественным образом может рассматриваться как подг руппа группы $G$.

Пусть теперь накрытие $g$ регулярно. Это означает, что подгруппа $g_{\#}\left(\pi_{1}(Z)\right) \subset$ $\pi_{1}(Y)$ является нормальным делителем. Следовательно, подгруппа

$$
H^{\prime}=g_{\#}\left(\pi_{1}(Z)\right) / p_{G_{\#}}\left(\pi_{1}(X)\right)
$$

является нормальньм делителем в группе

$$
\pi_{1}(Y) / p_{G_{\#}}\left(\pi_{1}(X)\right) .
$$

Но подгруппа $H^{\prime}$ соответствует именно подгруппе $H$ при естественном изоморфизме групп $\pi_{1}(Y) / p_{G_{\#}}\left(\pi_{1}(X)\right)$ и $G$.

Теорема 2.7. Орбитная проекиия $p_{G}: X \rightarrow Y=X / G$ действия абелевой группь порядка $n=p_{1} \cdots p_{k}$ представляется в виде композиции орбитных проекций действий групп $\mathbb{Z}_{p_{1}}, \ldots, \mathbb{Z}_{p_{k}}$.

ДоКАЗАТЕЛЬСТво проведем индукцией по числу $n(k)$. В абелевой групте $G$ порядка $p_{1} \cdots p_{k}$ имеется подгруппа $H$ порядка $p_{1} \cdots p_{k-1}$. Тогда орбитная проекция $p_{G}$ является композицией орбитных проекций $p_{H}$ и $p_{G / H}$, где на $X / H$ pacсматривается фактордействие групшы $\mathbb{Z}_{p_{k}}=G / H$.

В случае $G=\mathbb{Z}_{n}$, т.е. в случае периодического гомеоморфизма, теорема 2.7 превращается в теорему разложимости Баилдона [5; теорема 5].

Теорема 2.8. Орбитная проекиия $p_{G}: X \rightarrow Y=X / G$ действия разрешимой группы порядка $n=p_{1} \cdots p_{k}$ представляется в виде композиции орбитных проекций действий групп $\mathbb{Z}_{p_{1}}, \ldots, \mathbb{Z}_{p_{k}}$ в некотором порядке.

ДокАЗАТЕЛЬСтво. У разрешимой группы $G$ все факторы композиционного ряда абелевы. Отсюда следует, что проекция $p_{G}$ разлагается в композицию проекций действий абелевых групп, которые, в свою очередь, разлагаются в композицию проекций действий циклических групп простого порядка.

Теорема 2.9. Орбитная проекщия $p_{G}: X \rightarrow Y=X / G$ действия группьь порядка $n=p^{k}$, где $k \geqslant 2$, представляется в виде композиции орбитных проекций действий групп порядка $n=p^{m}$ и порядка $n=p^{k-m}$ для всякого $1 \leqslant m \leqslant k-1$.

ДокАЗАТЕЛЬСтво. В группе $G$ порядка $n=p^{k}$ имеется нормальный делитель $H$ порядка $n=p^{m}$ (для всякого $1 \leqslant m \leqslant k-1$ ) [13; 4.4.20.У], факторизация по которому и задает искомое разложение.

СлЕДСТВИЕ 2.10. Орбитная проекиия свободного действия разрешимой группь разлагается в композицию регулярных накрытий простых порядков.

СлЕДСтвИЕ 2.11. Регулярное накрытие нечетного порядка разлагается в композицию регулярных накрытий простых порядков.

ДокаЗАТЕЛЬСтво. Согласно теореме Фейта-Томпсона $[14 ;$ гл. 2, §5] всякая конечная группа нечетного порядка разрешима. 
СЛЕДСТВИЕ 2.12. Регулярное накрытие порядка $<60$ разлагается в композицию регулярных накрытий простых порядков.

ДоказАТЕльство. Всякая конечная группа порядка $<60$ разрешима $[15 ;$ гл. $11, \S 4$, задача 2].

СлЕДСТВИЕ 2.13. Каждое регулярное накрытие порядка $p^{k} q$, где $p$ u $q$ такие простые, что $p \geqslant q$, разлагается в композицию регулярного накрытия порядка $p^{k}$ и регулярного накрытия порядка $q$.

ДокАЗАтЕльство. Регулярное накрытие порядка $p^{k} q$ является орбитной проекцией свободного действия некоторой групшы $G$ поря дка $p^{k} q$. Если $p=q$, то группа $G$ имеет порядок $p^{k+1}$ и применима теорема 2.9. Если же $p>q$, то силовская $p$-подгруппа $G_{p}$ групшы $G$ имеет порядок $p^{k}$ и является нормальным делителем $[15$; гл. $11, \S 3$, пример 8]. Нормальная подгруппа $G_{p}$ определяет разложение заданного накрытия в композицию регулярного накрытия порядка $p^{k}$ и регулярного накрытия порядка $q$.

ТЕОРема 2.14. Если регулярное накрытие $f$ порядка $p^{k} q$, где $k \leqslant 2, p$ и $q$ такие простье, что $p>q$, разлагается в композицию регулярного накрьтия порядка q и регулярного накрыттия порядка $p^{k}$, то группа накрывающих преобразований накрытия $f$ абелева.

ДокАЗАТЕльство. Так как $p>q$, то силовская $p$-подгруппа $G_{p}$ группы $G$ (группы накрьвающих преобразований заданного накрытия) имеет порядок $p^{k}$ и является нормальным делителем $[15 ;$ гл. $11, \S 3$, пример 8]. Так как согласно условию $k \leqslant 2$, то группа $G_{p}$ абелева $[15 ;$ гл. $11, \S 4$, следствие 2$]$. Согласно теореме 2.6 разложение заданного накрытия $f$ определяет в группе $G$ некоторый нормальный делитель $H$ порядка $q$. Так как порядки подгрупп $G_{p}$ и $H$ взаимно просты, то эти подгруппы пересекаются только по единичному элементу. Из последнего вытекает, что элементы этих подгрупп коммутируют [15; гл. $11, \S 1$, предложение 1$]$.

Tеорема 2.15. Орбитная проекиия $p_{G}: X \rightarrow Y=X / G$ действия группьи порядка $n=p^{k} n_{1}$ представляется в виде композииии орбитной проекиии действия группь порядка $p^{k}$ и некоторого открытого отображсения порядка $\leqslant n_{1}$.

ДокАЗАтЕльство. Так как группа $G$ имеет порядок $p^{k} n_{1}$, то в ней имеется подгруппа порядка $p^{k}[13 ; 4.4 .6$. Т.У], факторизация по которой и задает искомое разложение.

СлЕДСТВИЕ 2.16. Каждое регулярное накрытие не простого порядка разпожимо.

\section{§3. Примеры и характеризация групп}

Теперь мы покажем, что препятствие из теоремы 2.3 не является полньм даже в самом простом случае ровно $n$-кратного открытого отображения, т.е. накрытия.

Теорема 3.1. Существует регулярное накрытие $f: S_{61} \rightarrow S_{2}$ порядка 60, которое непредставимо в виде нетривиальной композиции регулярных накрытий. 
ДокАЗАТЕльство. Рассмотрим регулярное накрытие $f: S_{61} \rightarrow S_{2}$ порядка 60 с группой накрывающих преобразований $A_{5}$. Как показал Джонс [16; таблица 5], имеется ровно 2016 таких неэквивалентных накрытий. Пусть $f$ представлено в виде композиции $f_{k} \circ \cdots \circ f_{1}$ накрытий, и пусть $f_{k}$ - регулярное накрытие. Рассмотрим разложение $f=f_{k} \circ h$, где $h=f_{k-1} \circ \cdots \circ f_{1}$. Согласно теореме 2.6 имеется такая подгруппа $H \subset A_{5}$, что $h=p_{H}$. Так как $f_{k}$ - регулярное накрытие, то согласно теореме 2.6 подгруппа $H$ является нормальным делителем, но в $A_{5}$ нет нетривиальных нормальных делителей.

Теорема 3.2. Существует регулярное накрытие $f: S_{13} \rightarrow S_{2}$ порядка 12 , всякое разложение $f=f_{i, 2} \circ f_{i, 1}$ которого на два открытых отображсения имеет один из следующих типов:

(1) Ord $f_{1,1}=2$, Ord $f_{1,2}=6 u f_{1,2}$ нерегулярно;

(2) Ord $f_{2,1}=3$, Ord $f_{2,2}=4$ u $f_{2,2}$ нерегулярно;

(3) Ord $f_{3,1}=4$, Ord $f_{3,2}=3 u f_{3,2}$ регулярно.

Причем в случаях (1) и (3) и только в этих случаях возможсно разложение на три открытых отобрахсения $f=f_{3} \circ f_{2} \circ f_{1}$. Разложсения (1) $u$ (3) соответствуют композициям $f_{1,1}=f_{1}, f_{1,2}=f_{3} \circ f_{2} u f_{3,1}=f_{2} \circ f_{1}, f_{3,2}=f_{3}$.

ДокАЗАтЕльство. Рассмотрим регулярное накрытие $f: S_{13} \rightarrow S_{2}$ порядка 12 с группой накрывающих преобразований $A_{4}$. Как показал Джонс [16; таблица 3], имеется ровно 200 таких неэквивалентных накрытий. Согласно теореме 2.3 разложение $f$ в композицию открытых отображений является разложением в композицию накрытий. Согласно теореме 2.6 всякое разложение $f$ в композицию двух нетривиальных накрытий соответствует собственной подгруппе $H$ группы $A_{4}$. В группе $A_{4}$ имеются [16; таблица 3$]$, [ $15 ;$ гл. $11, \S 5$, задача 2] собственные подгруппы только следующих типов:

$$
\begin{aligned}
& H=\mathbb{Z}_{2}, \quad\left|\mathbb{Z}_{2}\right|=2, \quad \mathbb{Z}_{2} \text { не является нормальной подгруппой; } \\
& H=\mathbb{Z}_{3}, \quad\left|\mathbb{Z}_{3}\right|=3, \quad \mathbb{Z}_{3} \text { не является нормальной подгруппой; } \\
& H=V_{4}, \quad\left|V_{4}\right|=4, \quad V_{4} \text { является нормальной подгруппой. }
\end{aligned}
$$

Эти три типа подгрупп соответствуют трем типам разложений в теореме. Так как накрытие $f_{3,1}$ регулярно, то согласно следствию 2.16 оно разлагается в композицию двух 2-кратных накрытий $f_{3,1}=f_{2} \circ f_{1}$. Разложение $f=\left(f_{3,2} \circ f_{2}\right) \circ f_{1}$ соответствует разложению типа (1).

СлеДСТвИЕ 3.3. Существует накрытие $f: S_{5} \rightarrow S_{2}$ порядка 4 , которое не разлагается в композицию двух нетривиальных открытых отображсений.

ДокАзАТЕльство. В качестве отображения $f$ возьмем $f_{2,2}$ из (2) теоремы 3.2. Если бы $f_{2,2}$ разлагалось, то мы имели бы $f_{2,2}=g \circ h$ и $h-$ двукратно. Тогда мы получили бы новое разложение $f=g \circ\left(h \circ f_{2,1}\right)$ в отображение кратности 6 и отображение кратности 2.

СлеДСтВИЕ 3.4. Существует накрытие $f: S_{7} \rightarrow S_{2}$ порядка 6, которое разлагается в композицию регулярных накрытий порядков 2 и 3, но не разлагается в композицию открытых отображсений порядков 3 и 2 и, следовательно, не является регулярным. 
ДоказАТельство. Разложение $f_{1,2}=f_{3} \circ f_{2}$ из теоремы 3.2 дает искомый пример. Отображение с указанными свойствами не может быть регулярньм согласно теореме 2.15 .

СледСтвиЕ 3.5. Существует регулярное накрытие $f: S_{13} \rightarrow S_{2}$ порядка 12, которое

(1) разлагается в композицию регулярного накрытия порядка 2 и накрытия порядка 6 , но не разлагается в композицию двух открытых отображений кратностей 6 и 2;

(2) разлагается в композицию регулярных накрытий кратностей 2,2 и 3 , но не разлагается в композицию открытых отображсений кратностей 2,3 и 2 или 3,2 и 2.

ДокАЗАТЕЛЬСтво. Искомым регулярным накрытием является накрытие теоремы 3.2 .

СлеДСТвиЕ 3.6. Существует регулярное накрытие $f: S_{7} \rightarrow S_{2}$ порядка 6 , которое представляется в виде композиции регулярных накрытий порядка 3 u 2, представляется в виде композиции регулярного накрытия порядка 2 и накрытия порядка 3 , но во втором разложсении второе накрытие не может быть регулярным.

ДоказАтельство. Рассмотрим регулярное накрытие $f: S_{7} \rightarrow S_{2}$ порядка 6 с группой накрываюших преобразований $D_{3}$. Как показал Джонс [16; таблица 1$]$, имеется ровно 60 таких неэквивалентных накрытий. Согласно теореме 2.3 разложение $f$ в композицию открытых отображений является разложением в композицию накрытий. Согласно теореме 2.6 всякое разложение $f$ в композицию двух нетривиальных накрытий соответствует собственной подгруппе $H$ группы $D_{3}$. В группе $D_{3}$ есть нормальный делитель порядка 3 и нет нормального делителя порядка $2[15 ;$ гл. 11, § 1 , пример 16].

При изучении поверхности $S_{g}$ важное значение имеет группа

$$
\Pi_{g}=\pi_{1}\left(S_{g}\right)=\left\{a_{1}, b_{1}, \ldots, a_{g}, b_{g}:\left[a_{1}, b_{1}\right] \cdots\left[a_{g}, b_{g}\right]=e\right\},
$$

где $[\cdot, \cdot]$ означает коммутатор.

ТЕОРема 3.7. Всякая группа $G$ с $k$ образующими является гомоморфныцм образом группь $\Pi_{g}$ при $g \geqslant k$. Всякая группа $G$ с $k$ образующими является группой накрывающих преобразований некоторого регулярного накрытия поверхности $S_{g}$ при $g \geqslant k$. Всякая группа $G$ порядка $n c k$ образующими свободно действует на $S_{h}$ при $h=n(g-1)+1 u g \geqslant k$.

ДокАЗАТЕЛЬСтво. Для всякого $i=1, \ldots, g$ фиксируем целое число $k_{i}$ (например, положим $k_{i}=0$ ). Отображение $a_{i} \mapsto a_{i}, b_{i} \mapsto a_{i}^{k_{i}}$ задает гомоморфизм группы П $\Pi_{g}$ в свободную группу $F_{g}$ с $g$ образуюшими. Всякая группа с $k$ образуюшими является гомоморфным образом свободной группы $F_{g}$ при $g \geqslant k$.

Пусть $G$ - группа с $k$ образующими, а $\varphi: \Pi_{g} \rightarrow G$ - некоторый эпиморфизм. Подгруппа $H=\operatorname{ker} \varphi \subseteq \pi_{1}\left(S_{g}\right)$ является нормальньм делителем. Поэтому накрытие, соответствуюшее этой подгруппе, регулярно и группой его накрываюших преобразований является группа $\pi_{1}\left(S_{g}\right) / H=G$. 
Согласно только что доказанному утверждению сушествует регулярное накрытие $f: S \rightarrow S_{g}$ с заданной группой накрывающих преобразований $G$. Так как группа $G$ конечна, то поверхность $S$ замкнута и компактна и $\chi(S)=|G| \cdot \chi\left(S_{g}\right)$, т.е. $2-2 h=n \cdot(2-2 g)$.

Отсюда вытекает следуюший регулярный вариант теоремы Хаймеса-Штёккеpa.

СлеДСТВИЕ 3.8. Для замкнутых ориентируемых поверхностей $S_{h}, S_{g} u$ числа $n \geqslant 2$ следующие условия әквивалентны:

(1) существует регулярное накрытие $f: S_{h} \rightarrow S_{g}$ с группой накрывающих преобразований $\mathbb{Z}_{n} ;$

(2) существует накрытие $f: S_{h} \rightarrow S_{g}$ кратности $n$;

(3) $h-1=n(g-1)$.

ЛЕмма 3.9. При $n \geqslant 5$ всякая собственная подгруппа $A_{n}$ имеет индекc $\geqslant n$.

ДоказАтельство. Пусть $H$ - собственная подгруппа $A_{n}$. На множестве левых смежных классов группы $A_{n}$ по подгруппе $H$ определено левое действие группы $A_{n}$. Так как группа $A_{n}$ при $n \geqslant 5$ простая [15; гл. $\left.11, \S 5\right]$, то гомоморфизм $A_{n} \rightarrow S\left(A_{n} / H\right)$ является мономорфизмом. Отсюда следует неравенство $n ! / 2 \leqslant m !$, где $m=\left|A_{n} / H\right|=\left|A_{n}: H\right|$, равносильное требуемому.

СлЕДСТВИЕ $3.8^{\prime}$. В случае $g \geqslant 2$ условия (1)-(3) следствия 3.8 әквивалентны условию:

(4) существует накрытие $f: S_{h} \rightarrow S_{g}$ кратности $n$, которое не разлагается в композичию двух нетривиальных открытых отображсений.

ДокАЗАТЕЛьСтво. Импликация $(4) \Rightarrow(2)$ очевидна.

$(3) \Rightarrow(4)$. При $n=2,3$ все справедливо, так как эти значения $n$ являются простыми.

Группа $A_{n}$ порождена двумя элементами. Согласно теореме 3.7 существует регулярное накрытие $F: S_{(n ! / 2)(g-1)+1} \rightarrow S_{g}$ с группой накрывающих преобразований $A_{n}$.

Будем считать, что $A_{n-1}$ вложена в $A_{n}$ в виде подгруппы, оставляющей на месте символ $n$. Подгруппа $A_{n-1}$ имеет индекс $n$, поэтому индуцированное накрытие $f: S_{(n ! / 2)(g-1)+1} / A_{n-1} \simeq S_{n(g-1)+1} \rightarrow S_{g}$ имеет порядок $n$.

Согласно лемме 3.9 при $n \geqslant 5$ подгруппа $A_{n-1}$ является максимальной в $A_{n}$. В максимальности подгруппы $A_{3}$ в $A_{4}$ можно убедиться по таблице 3 из [16]. Поэтому согласно теореме 2.6 накрытие $f$ неразложимо.

СлеДСТВИЕ 3.10. Существует регулярное накрытие $f: S_{361} \rightarrow S_{2}$ порядка 360 , во всяком разложении которого в композицию нетривиальньх открытых отображсений кратность последнего отображсения является непроствим числом.

ДокАЗАтЕЛьство. Согласно теореме 3.7 существует регулярное накрытие

$$
f: S_{361} \rightarrow S_{2}
$$


с группой накрывающих преобразований $A_{6}$.

Согласно теореме 2.6 для всякого разложения $f=f_{k} \circ \cdots \circ f_{1}$ существует такая подгруппа $H \subset A_{6}$, что $f_{k-1} \circ \cdots \circ f_{1}=p_{H}$. Согласно теореме 2.3 кратность отображения $f_{k}$ равна индексу $\left|A_{6}: H\right|$, который $\geqslant 6$ согласно лемме 3.9 , а согласно теореме Лагранжа $[15 ;$ гл. $4, \S 4]$ делит порядок группы $\left|A_{6}\right|=6$ !. Полученные два условия показывают, что порядок Ord $f_{k}$ не может быть простым числом.

Tеорема 3.11. Существует регулярное накрытие $f: S_{121} \rightarrow S_{2}$ порядка 120, которое представимо в виде композиции регулярных накрытий порядка 2 и 60, но непредставимо в виде композиции открытых отображений порядка 60 и 2.

ДокАЗАТЕльство. Групша $\mathrm{SL}_{2}\left(\mathbb{Z}_{5}\right)$ имеет порядок 120 и содержит единственный нетривиальный нормальный делитель - центр $Z\left(\mathrm{SL}_{2}\left(\mathbb{Z}_{5}\right)\right)[14 ;$ гл. 2, начало доказательства 5.3]. Следовательно, эта группа не содержит подгрупп порядка 60. Группа $\mathrm{PSL}_{2}\left(\mathbb{Z}_{5}\right)=\mathrm{SL}_{2}\left(\mathbb{Z}_{5}\right) / Z\left(\mathrm{SL}_{2}\left(\mathbb{Z}_{5}\right)\right) \simeq A_{5}[15 ;$ гл. $11, \S 5$, задача 3 или 4$]$ порождена двумя элементами. Отсюда вытекает, что и группа $\mathrm{SL}_{2}\left(\mathbb{Z}_{5}\right)$ порождена любыми двумя элементами, проекции которых порождают групп $\mathrm{PSL}_{2}\left(\mathbb{Z}_{5}\right)$. Cогласно теореме 3.7 сушествует регулярное накрытие $f: S_{121} \rightarrow S_{2}$ порядка $120 \mathrm{c}$ группой накрьвающих преобразований $\mathrm{SL}_{2}\left(\mathbb{Z}_{5}\right)$. Группа $\mathrm{SL}_{2}\left(\mathbb{Z}_{5}\right)$ содержит нормальный делитель порядка 2 (центр), но не содержит подгруппу порядка 60 , что согласно теореме 2.6 завершает доказательство.

Для всякой конечнопорожденной группы $G$ определен инвариант

$$
g(G)=\min \left\{g: \text { группа } G \text { является гомоморфным образом группы } \Pi_{g}\right\} .
$$

Легко видеть, что $g(G)=1$ тогда и только тогда, когда группа $G$ является абелевой и порождена двумя элементами. Для всякой неабелевой группы $G$ с двумя образующими $g(G)=2$. Для всякой абелевой групшы $G$ с $k$ образуюшими справедливо равенство $g(G)=[(k+1) / 2]$, где [· $]$ означает целую часть. В работах Фробениуса, Медных и Джонса [17], [18], [16] даются точные формулы для числа $\Phi_{g}(G)$ эпиморфизмов $\Pi_{g}$ на $G$ (числа $n_{g}(G)$ неэквивалентных накрытий $S_{g}$ с заданной группой накрываюших преобразований $G$ ), однако анализ этих формул для получения неравенства $n_{g}(G) \geqslant 1$ в общем случае представляет значительные трудности. Видимо, в общем случае инвариант $g(G)$ вычислить довольно сложно. Имеется много работ, в которых рассматривается "обратная" задача - по роду $h$ поверхности оценивается порядок эффективно (не обязательно свободно) действующей на ней группы $[8 ; \S 4.15]$.

ТЕорема 3.12. Для конечной группы $G$ следующие условия әквивалентны:

(1) $G$ - не простая группа;

(2) всякое регулярное накрытие $f: X \rightarrow Y$ связных пространств с группой накрьвающих преобразований $G$ разлагается в композицию двух нетривиальных регулярных накрытий;

(3) всякое регулярное накрытие $f: S_{h} \rightarrow S_{g}$ с группой накрывающих преобразований $G$ разлагается в композицию двух нетривиальных регулярных накрытий; 
(4) существует регулярное накрытие $f: X \rightarrow Y$ связных пространств с группой накрьвающих преобразований $G$, которое разлагается в композичию двух нетривиальных регулярных накрытий.

ДокАЗАтЕльство. $(1) \Rightarrow(2)$. Если $G$ не простая группа, то сушествует нормальный делитель $H \subset G$ и орбитная проекция $p_{G}: X \rightarrow Y=X / G$ разлагается в композицию двух орбитных проекций $X \rightarrow Z=X / H \rightarrow Y=X / G=Z /(G / H)$.

Импликация $(2) \Rightarrow(3)$ очевидна.

Импликация $(3) \Rightarrow(4)$ содержится в теореме 3.7 .

$(4) \Rightarrow(1)$. Пусть некоторое регулярное накрытие $p: X \rightarrow Y$ с группой накрываюших преобразований $G$ представляется в виде композиции $X \rightarrow Z \rightarrow Y$ двух регулярных накрытий. Согласно теореме 2.6 это разложение соответствует некоторой подгрупше $H \subset G$. Так как второе накрытие $Z \rightarrow Y$ тоже регулярно, то подгруппа $H$ является нормальным делителем, т.е. группа $G$ не является простой.

Теорема 3.13. Для конечной группь $G$ следующие условия әквивалентны:

(1) $G$ - разрешимая группа;

(2) всякое регулярное накрытие $f: X \rightarrow Y$ связных пространств с группой накрывающих преобразований $G$ разлагается в композичию регулярных накрытий простых порядков;

(3) всякое регулярное накрытие $f: S_{h} \rightarrow S_{g}$ с группой накрывающих преобразований $G$ разлагается в композицию регулярных накрытий простых порядков;

(4) существует регулярное накрытие $f: X \rightarrow Y$ связных пространств с группой накрьвающих преобразований $G$, которое разлагается в композицию регулярных накрьтий простых порядков.

ДокАЗАТЕльство. $(1) \Rightarrow(2)$. Разложимость является в точности утверждением теоремы 2.8 .

Импликация $(2) \Rightarrow(3)$ очевидна.

Импликация $(3) \Rightarrow(4)$ содержится в теореме 3.7 .

$(4) \Rightarrow(1)$. Пусть $n=p_{1} \cdots p_{k}$. Доказательство проведем индукцией по числу $k$. Если $k=1$, то $G=\mathbb{Z}_{p_{1}}$ - разрешимая группа. Пусть $k \geqslant 2$ и $f=f_{k} \circ \cdots \circ f_{1}$, где $f_{k}$ - регулярное накрытие порядка $p_{i_{k}}$. Согласно теореме 2.6 существует такой нормальный делитель $H \subset G$, что $G / H=\mathbb{Z}_{p_{i_{k}}}$ и орбитная проекция $p_{H}=$ $f_{k-1} \circ \cdots \circ f_{1}$ является композицией регулярных накрытий простых порядков. По индуктивному предположению группа $H$ разрешима, и так как факторгруппа $G / H$ абелева, то группа $G$ также разрешима.

\section{§4. Регуляризация}

Со всяким регулярным накрытием связана группа накрывающих преобразований. Ниже мы показываем, что с общим накрытием порядка $n$ естественным образом связаны некоторая конечная группа $G$ и ее подгруппа $H$ индекса $n$, не содержащая нетривиальный нормальный делитель $G$.

ТЕОРема 4.1. Для всякого накрытия $f: X \rightarrow Y$ порядка $n$ существуют регулярные накрытия $\widetilde{f}: \widetilde{X} \rightarrow Y$ порядка $\widetilde{n}$, делящегося на $n$ и делящего $n !, u$ $p_{f}: \widetilde{X} \rightarrow X$ mакие, что $\widetilde{f}=f \circ p_{f}$ и всякое регулярное накрытие $\widehat{f}: \widehat{X} \rightarrow Y$, 
факторизующееся через накрытие $f$, факторизуется через накрытие $\widetilde{f}$, т.е. uз $\widehat{f}=f \circ g$ для некоторого накрытия $g: \widehat{X} \rightarrow X$ вытекает существование такого накрытия $p_{\widetilde{f}}^{\widehat{f}}: \widehat{X} \rightarrow \widetilde{X}$, что $g=p_{f} \circ p_{\widetilde{f}}^{\widehat{f}}$.

ДокАЗАТЕЛЬСтво. Выделим в пространстве $X$ некоторую точку $x_{0}$, и пусть $y_{0}=f\left(x_{0}\right)$. В группе $\pi_{1}\left(Y, y_{0}\right)$ рассмотрим подгруппу $H=f_{*}\left(\pi_{1}\left(X, x_{0}\right)\right)$ индекca $n$. В $[13 ; 4.3 .10 . Т]$ описан максимальный нормальный делитель $\widetilde{H}$, содержащийся в данной подгруппе $H$ группы $G$. Из теоремы Пуанкаре [19; гл. III, $\S 8]$ вытекает, что если $H$ имеет в $G$ конечный индекс $n$, то $\widetilde{H}$ имеет конечный индекс $\leqslant n^{n}$. Из рассмотрения группы монодромии $[20 ; \S 58]$ накрытия, соответствующего подгруппе $H$, вытекает требуемое более сильное условие на индекс $\widetilde{H}$ в $G$. Накрытие пространства $\left(Y, y_{0}\right)$, соответствуюшее нормальному делителю $\widetilde{H}$, и является искомьм минимальным регулярньм накрытием, факторизующимся через накрытие $f$.

Пусть теперь $\widehat{f}: \widehat{X} \rightarrow Y$ - такое регулярное накрытие, что $\widehat{f}=f \circ g$ для некоторого накрытия $g: \widehat{X} \rightarrow X$. Выберем какую-нибудь точку $\widehat{x}_{0} \in g^{-1}\left(x_{0}\right)$. Так как накрытие $\widehat{f}$ регулярно и $\widehat{f}=f \circ g$, то $\widehat{H}=\widehat{f}_{*}\left(\pi_{1}\left(\widehat{X}, x_{0}\right)\right)$ является нормальной подгруппой, которая содержится в подгруппе $H$. Отсюда вытекает включение $\widehat{H} \subseteq \widetilde{H}$. Последнее включение влечет сушествование такого отображения $p_{\widetilde{f}}^{\widehat{f}}:\left(\widehat{X}, \widehat{x}_{0}\right) \rightarrow\left(\widetilde{X}, \widetilde{x}_{0}\right)$, что $\widehat{f}=\widetilde{f} \circ p_{\widetilde{f}}^{\widehat{f}}$, т.е. $f \circ g=f \circ p_{f} \circ p_{\widetilde{f}}^{\widehat{f}}$. Отображения $g$ и $p_{f} \circ p_{\widehat{f}}^{\widehat{f}}$ являются двумя поднятиями в $X$ отображения $\widehat{f}$. Так как при этом $g\left(\widehat{x}_{0}\right)=x_{0}=p_{f}\left(\widetilde{x}_{0}\right)=p_{f}\left(p_{\widetilde{f}}^{\widehat{f}}\left(\widehat{x}_{0}\right)\right)$ и пространство $\widehat{X}$ связно, то эти поднятия $g$ и $p_{f} \circ p_{\widehat{f}}^{\widehat{f}}$ совпадают на всем пространстве $\widehat{X}$.

ЗАмЕчАНИЕ 4.2. Вопрос разложимости произвольного накрытия $f$ в композицию двух накрытий является довольно сложным. Построенное регулярное накрытие $\widetilde{f}$ сводит вопрос о разложениях накрытия $f$ к вопросу о разложениях накрытия $\widetilde{f}$ большего порядка, которое, однако, уже является регулярным и поэтому разложения которого описываются теоремой 2.6. Построенное регулярное накрытие $\widetilde{f}$ "минимально", поэтому группа накрьвающих преобразований накрытия $\tilde{f}$ (это есть группа монодромии накрытия $f[20, \S 58]$ ) и подгруппа (индекса $n$ ) накрываюших преобразований накрытия $p_{f}$ однозначно определяются накрытием $f$.

Продемонстрируем один пример применения теоремы.

Напомним, что под слабо соленоидальной последовательностью (соленоидальной последовательностью) замкнутых многообразий понимается обратная последовательность замкнутых многообразий $X_{n}$, в которой связывающие отображения $f_{m}^{n}: X_{n} \rightarrow X_{m}$ являются (регулярными) накрытиями. Обратньй предел $X_{\infty}$ назьвается слабо соленоидальным пространством (соленоидальныцм пространством). Маккорд показал [21], что всякое соленоидальное пространство топологически однородно. Шори [22] и Роджерс, Толлефсон [23] построили примеры топологически неоднородных слабо соленоидальных пространств. Пример Роджерса-Толлефсона 2-кратно накрывается соленоидальным пространством. В некотором смысле это есть обший случай.

ТЕОРема 4.3. Для всякого слабо соленоидального пространства $Y_{\infty}$ существуют соленоидальное пространство $X_{\infty}$ и расслоение Гуревича $p_{\infty}: X_{\infty} \rightarrow Y_{\infty}$ со свойством единственности накрьвающего пути. 
ДокАЗАТЕЛЬСТво. Спектр $\left\{X_{n}, g_{m}^{n}\right\}$ построим индукцией по $n$. Положим $X_{1}=Y_{1}$. Для накрытия $f_{1}^{n}: Y_{n} \rightarrow Y_{1}$ рассмотрим минимальное регуляризуюшее накрытие $\tilde{f}_{1}^{n}: \widetilde{Y}_{n} \rightarrow Y_{1}$ из теоремы 4.1. Положим $X_{n}=\widetilde{Y}_{n}$. Из минимальности $\widetilde{f}_{1}^{n-1}$ и равенства $f_{1}^{n}=f_{1}^{n-1} \circ f_{n-1}^{n}$ вытекает сушествование такого накрытия $g_{n-1}^{n}=p_{\tilde{f}_{1}^{n-1}}^{\widetilde{f}_{n}^{n}}: \widetilde{Y}_{n}=X_{n} \rightarrow \widetilde{Y}_{n-1}=X_{n-1}$, что $f_{n-1}^{n} \circ p_{f_{1}^{n}}=p_{f_{1}^{n-1}} \circ g_{n-1}^{n}$. Coгласно теореме 2.6 все связывающие отображения $g_{m}^{n}$ суть регулярные накрытия, поэтому обратньй предел $X_{\infty}$ является соленоидальным пространством.

Отображения $\left\{p_{f_{1}^{n}}: X_{n} \rightarrow Y_{n}\right\}$ являются регулярными накрытиями и задают отображение спектра $\left\{X_{n}, g_{m}^{n}\right\}$ в спектр $\left\{Y_{n}, f_{m}^{n}\right\}$.

Действительно, согласно теореме 2.6 все накрытия $p_{f_{1}^{n}}$ регулярнг; равенство $f_{n-1}^{n} \circ p_{f_{1}^{n}}=p_{f_{1}^{n-1}} \circ g_{n-1}^{n}$ означает, что $\left\{p_{f_{1}^{n}}\right\}_{n=1}^{\infty}$ является отображением спектров. Предельное отображение $p_{\infty}: X_{\infty} \rightarrow Y_{\infty}$ и есть расслоение Гуревича со свойством единственности накрьвающего пути (как обратный предел накрытий).

\section{§5. Разветвленные накрытия}

Построенные выше препятствия к разложимости накрытия в композицию имеют глобальный характер. Теперь мы обсудим чисто локальное препятствие.

Напомним [6; X, (7.3)], [24]-[27], что открытое отображение $f: S_{h} \rightarrow S_{g}$ конечной кратности $n$ является локальным гомеоморфизмом во всех точках поверхности $S_{h}$, кроме конечного сингулярного подмножества. Образ $B_{f}$ этого сингулярного множества назьвается множеством ветвления. В окрестности всякой точки $x \in S_{h}$ отображение $f$ эквивалентно комплексной функции $z \mapsto z^{n_{x}}$ для некоторого $n_{x} \geqslant 1$, и $x$ соответствует нулю в $\mathbb{C}$. Для всякой точки $y \in S_{g}$ справедливо равенство $n=\sum_{x \in f^{-1}(y)} n_{x}$. Следовательно, всякой точке $y \in S_{g}$ соответствует такое разбиение $A(y)=\left[n_{1}, \ldots, n_{\mu_{y}}\right]$ числа $n$, что $f^{-1}(y)=\left\{x_{1}, \ldots, x_{\mu_{y}}\right\}$ и $n_{i}=n_{x_{i}}$. Множество точек $y \in S_{g}$, для которых $A(y)$ не является тривиальньм разбиением $[1, \ldots, 1]$ числа $n$, и есть в точности множество ветвления $B_{f}$. Семейство разбиений $\mathscr{D}(f)=\{A(y)\}_{y \in B_{f}}$ называется массивом ветвлений. Массив ветвлений дает новое препятствие к разложимости.

Ясно, что если $y \in Y$ - точка ветвления композиции $f=g \circ h$, где $h: X \rightarrow Z$ и $g: Z \rightarrow Y$ - разветвленные накрытия, то или $y$ - точка ветвления разветвленного накрытия $g$, или по крайней мере одна из точек $g^{-1}(y)$ является точкой ветвления разветвленного накрытия $h$.

Пусть $h: X \rightarrow Z$ и $g: Z \rightarrow Y$ - разветвленные накрытия порядков $n_{1}$ и $n_{2}$ соответственно, $f=g \circ h$ - композиция, а $B_{f}=\left\{y_{1}, \ldots, y_{m}\right\}$ - множество всех точек ветвления композиции. Пусть $\left[n_{1,1}, \ldots, n_{1, q_{1}}\right], \ldots,\left[n_{m, 1}, \ldots, n_{m, q_{m}}\right]-$ массив ветвления $f$, а $z_{i, 1}, \ldots, z_{i, t_{i}}$ - все прообразы точки $y_{i}$ при отображении $g$, которое имеет в них ветвления порядков $k_{i, 1}, \ldots, k_{i, t_{i}}$ соответственно. Сумма порядков ветвления равна порядку $g: k_{i, 1}+\cdots+k_{i, t_{i}}=n_{2}$ для всех $i=1, \ldots, m$. Пусть $x_{i, j, 1}, \ldots, x_{i, j, v_{i, j}}$ - все прообразы точки $z_{i, j}$ при отображении $h$, которое имеет в них ветвления порядков $r_{i, j, 1}, \ldots, r_{i, j, v_{i, j}}$ соответственно. Сумма порядков ветвления равна порядку $h: r_{i, j, 1}+\cdots+r_{i, j, v_{i, j}}=n_{1}$ для всех $i=1, \ldots, m, j=1, \ldots, t_{i}$. Отображение $f=g \circ h$ имеет ветвление в точке $x_{i, j, l}$ порядка $r_{i, j, l} \cdot k_{i, j}$. Следова- 
тельно, массивом ветвления точки $y_{i}$ является набор чисел

$$
\left[r_{i, j, l} \cdot k_{i, j}\right]_{l=1, \ldots, v_{i, j}}
$$

Представимость заданного разбиения $\left[n_{i, 1}, \ldots, n_{i, q_{i}}\right]$ числа $n=n_{1} n_{2}$ в виде произведения $(\mathrm{m})$ является сильным локальным препятствием разложимости заданного разветвленного накрытия в виде композиции разветвленных накрытий. Приведем примеры.

СлЕДСТВИЕ 5.1. Открытое отображение $f: S_{h} \rightarrow S_{g}$ порядка 4, массив ветвления которого содержит разбиение [3,1], непредставимо в виде нетривиальной композичии открытых отображений.

Примером такого отображения является отображение сферы Римана, задаваемое многочленом $z^{4}+z^{3}$.

ЗАмЕчаниЕ 5.2. Известная теорема Кнезера [8; $§ 3.3]$, [28] утверждает, что если непрерывное отображение поверхностей $f: S_{h} \rightarrow S_{g}$ имеет положительную степень $n$, то $h \geqslant n(g-1)+1$. Неравенство Кнезера налагает сильное ограничение на поверхность $Z$, через которую факторизуется заданное отображение $f$ (если такая факторизация возможна). Так, отображение сферы в сферу может факторизоваться только через сфиеру.

ЗАмЕчАнИЕ 5.3. Более сильное ограничение на поверхность $Z$, через которую факторизуется заданное отображение $f$ (если такая факторизация возможна), и на индексы ветвления точек ветвления отображений разложения налагает формула Римана-Гурвица [6; X, (7.3)], [24]-[32].

СледСтвиЕ 5.4. Отображение сферь Римана, задаваемое многочленом $f(z)=z^{5}(z-a)^{4} p_{6}(z)+\lambda z^{5}\left(35 z^{3}-120 a z^{2}+140 a^{2} z-56 a^{3}\right)=z^{5}(z-a)^{4} p_{6}(z)+$ $\lambda(z-a)^{4}\left(35 z^{4}+20 a z^{3}+10 a^{2} z^{2}+4 a^{3} z+a^{4}\right)-\lambda a^{8}$, где $p_{6}(z)$ - такой многочлен степени 6 , что $а p_{6}(0) \neq 56 \lambda, a_{6}(a) \neq-70 \lambda$ и $а \neq 0$, непредставимо в виде нетривиальной композиции открытых отображений.

ДоказАтЕльство. Пусть $f=g \circ h$, где $h: S_{0} \rightarrow S_{0}$ и $g: S_{0} \rightarrow S_{0}$-открытые отображения кратностей $\geqslant 2$. Согласно теореме 2.3 одно из этих отображений имеет кратность 3 , а другое - кратность 5 .

Точка $\infty$ является точкой ветвления $f$ с массивом ветвления [15]. Из заданных условий вытекает, что точка 0 является точкой ветвления с массивом ветвления $[5, \ldots]$. Как число 15 , так и число 5 единственньм образом разлагаются в произведение двух таких чисел $\alpha$ и $\beta$, что $\alpha \leqslant 5$ и $\beta \leqslant 3$. Следовательно, отображение поря дка 5 имеет по крайней мере 2 точки ветвления порядка 5 . Из формулы Римана-Гурвица вытекает, что отображение кратности 5 имеет только эти две точки ветвления. Тогда из формулы $(\mathrm{m})$ вытекает, что в массиве ветвления любой точки ветвления композиции $f=g \circ h$ числа кратны 5 или не превосходят 3 . Однако из заданных условий вытекает, что при $\lambda \neq 0$ точка $-\lambda a^{8}$ является точкой ветвления $f$ с массивом ветвления $[4, \ldots]$, а при $\lambda=0$ точка 0 является точкой ветвления $f$ с массивом ветвления $[5,4, \ldots]$. 
СледСтвиЕ 5.5. Отображсение $f$ сферы Римана, задаваемое многочленом $f(z)=z^{9} p_{6}(z)$, где $p_{6}(z)$ - такой многочлен степени 6 , что $p_{6}(0) \neq 0$, представимо в виде композиции открытых отображений кратностей 5 и 3 тогда и только тогда, когда многочлен $p_{6}(z)$ является кубом некоторого многочлена второй степени.

ДокАЗАтЕльство. Отображение $f(z)=z^{9}\left(A z^{2}+B z+C\right)^{3}$ обладает искомым разложением. Достаточно положить $g(z)=z^{3}$ и $h(z)=z^{3}\left(A z^{2}+B z+C\right)$.

Пусть $f=g \circ h$, где $h: S_{0} \rightarrow S_{0}$ и $g: S_{0} \rightarrow S_{0}$ - открытые отображения и $\operatorname{Ord} h=5$, Ord $g=3$.

Из заданного условия вытекает, что точка 0 является точкой ветвления с массивом ветвления $[9, \ldots]$. Число 9 единственным образом разлагается в произведение двух таких чисел $\alpha$ и $\beta$, что $\alpha \leqslant 5$ и $\beta \leqslant 3$. Следовательно, отображение порядка 3 имеет в точке 0 ветвление порядка 3 . Тогда из формулы $(\mathrm{m})$ вытекает, что массивом ветвления точки 0 при композиции $f=g \circ h$ будет $[9,6]$ или $[9,3,3]$ в зависимости от того, каков массив ветвления точки $g^{-1}(0)$ при отображении $h$. Это означает, что композиция $f$ имеет вид $\widetilde{A} z^{9}(z-a)^{6}$ в первом случае и $\widetilde{A} z^{9}\left(z-a_{1}\right)^{3}\left(z-a_{2}\right)^{3}$ во втором случае.

ЗАмЕчАНИЕ 5.6. Неразветвленные накрытия порядка $n$ над пространством $Y$ описываются представлениями группы $\pi_{1}(Y)$ в симметрической группе от $n$ символов, т.е. в группе $\Sigma_{n}[20 ; \S 58]$. Это означает, что любое свойство накрытия, в частности разложимость в композицию, может быть описано в терминах соответствуюшего представления. Для примера укажем, что если представление накрытия порядка 4 содержит перестановку $(1,2,3)(4)$, то это накрытие не разлагается в композицию двух накрытий.

ЗАмечАние 5.7. Разветвленные накрытия порядка $n$ над поверхностью $S$ с множеством ветвления $B \subset S$ описываются представлениями групшы $\pi_{1}(S \backslash B)$ в симметрической группе $\Sigma_{n}[24]-[27]$. Это означает, что разложимость разветвленного накрытия в композицию также может быть описана в терминах соответствуюшего представления. Для примера укажем, что следствие 5.1 может быть получено и таким алгебраическим способом. Именно таким образом показывается, что формула Римана-Гурвица не дает полное препятствие для реализуемости над сферой или проективным пространством абстрактного массива ветвления, что дает дополнительное препятствие к разложимости разветвленных накрытий над сфиерой $[24 ; 3.5],[26 ; 5.3,5.6,5.7,6.3,6.4]$ и проективным пространством $[24 ; 3.6]$, $[26 ; 5.9]$.

ЗАмЕчАнИЕ 5.8. В работах [24]-[27] строятся различные примитивные разветвленные накрытия. Вместе с классификационными теоремами Габаи-Казеса [33] и Натанзона [34], [35], Протопопова [36], Хованского-Здравковской [37] эти накрытия позволяют доказать разложимость некоторых классов примитивных разветвленных накрытий. Всякое непримитивное разветвленное накрытие представимо в виде композиции примитивного разветвленного накрытия и обычного накрытия $[27 ; 5.19]$. Композиция примитивных разветвленных накрытий является примитивным разветвленным накрытием, а нетривиальное неразветвленное накрытие (в том числе и простое) не является примитивным. Следовательно, простота и примитивность неразветвленного накрытия являются несравнимыми понятиями. 
ЗАмЕЧАНИЕ 5.9. Хованский и Здравковска с помощью групп кос получили результаты о разложимости полиномиальных отображений, которые не определяются массивом ветвления [37; примеры 1 и 2].

ЗАмечАние 5.10. Согласно теореме Сиеклуцкого-Кржемпека аналог формулы (М) для разложений в композицию неоткрытых отображений не может иметь место. Однако если в “неоткрытом" разложении предполагать, что промежуточные пространства являются поверхностями, то с помощью неравенства РиманаГурвица-Кнезера-Хопфа [31], [38] можно получить довольно сильные условия на пространства и отображения разложения. Мультипликативность в этом случае является проявлением обычной мультипликативности степени отображения.

ЗАмЕчАниЕ 5.11. В работах [39], [40] предложены препятствия к представлению заданного отображения (полинома [41]) в виде итерации одного и того же произвольного (мероморфного [42]) отображения.

ЗАмЕчАнИЕ 5.12. Так как открытый конечнократный образ $n$-многообразия при $n \geqslant 3$ не всегда является $n$-многообразием, то в многомерном случае дословное повторение рассуждений настоящей работы невозможно. Однако результаты и методы работ [43]-[54] позволяют получить некоторые многомерные аналоги формулы (M).

Авторам приятно выразить свою благодарность А. Ю. Ольшанскому и И. А. Чубарову за полезные беседы.

ПРИМЕЧАНИЕ ПРИ КОРРЕКТУРЕ. ЗаДаче описания всех полиномиальных разложений данного полиномиального отображения сферы Римана в себя посвящена обширная литература [55]-[62]. Так называемая первая теорема Ритта утверждает [55], что для любых двух разложений полинома $f$ в композицию неразложимых полиномов $f=f_{k} \circ \cdots \circ f_{1}=g_{m} \circ \cdots \circ g_{1}$ обязательно $k=m$ и $\operatorname{deg} f_{i}=\operatorname{deg} g_{\sigma(i)}$ для некоторой перестановки $\sigma$. Подчеркнем, что согласно теореме 3.2 для поверхностей рода $\geqslant 2$ единственности числа отображений в композиции нет даже в случае регулярного накрытия.

\section{Список литературы}

1. Borsuk K., Molski R. On a class of continuous mappings // Fund. Math. 1957. V. 45. № 1. P. 84-98.

2. Sieklucki K. On superposition of simple mappings // Fund. Math. 1960. V. 48. № 2 . P. 217-228.

3. Krzempek J. Compositions of simple maps // Fund. Math. 1999. V. 162. P. 149-162.

4. Dydak J. On elementary maps // Colloq. Math. 1974. V. 31. № 1. P. 67-69.

5. Baildon J. D. Open simple maps and periodic homeomorphisms // Proc. Amer. Math. Soc. 1973. V. 39. № 2. P. 433-436.

6. Whyburn G. T. Analitic topology. Providence, RI: Amer. Math. Soc., 1963. (Amer. Math. Soc. Colloq. Publ. V. 28.)

7. Heimes R., Stöcker R. Coverings of surfaces // Arch. Math. 1978. V. 30. № 2. P. 181-187.

8. Цишанг X., Фогт Э., Колдевай X.-Д. Поверхности и разрывные группы. М.: Наука, 1988.

9. Спеньер Э. Алгебраическая топология. М.: Мир, 1971.

10. Lubkin S. Theory of covering spaces // Trans. Amer. Math. Soc. 1962. V. 104. № 2 . P. 205-238.

11. Fox R. H. Shape theory and covering spaces // Lecture Notes in Math. 1974. V. 375. P. 71-90. 
12. Jaworowski J. W. On simple regular mappings // Fund. Math. 1958. V. 45. № 2 . P. 119-129.

13. Ляпин E.C., Айзенштат A.Я., Лесохин M.M. Упражнения по теории групп. М.: Наука, 1967.

14. Бахтурин Ю. А. Основные структуры современной алгебры. М.: Наука, 1990.

15. Винберг Э. Б. Курс алгебры. М.: Факториал, 1999.

16. Jones G. A. Enumeration of homomorphisms and surface-coverings // Quart. J. Math. Oxford (2). 1995. V. 46. P. 485-507.

17. Frobenius G. Über Gruppencharaktere // Sitzungsber. Königlich Preuss. Akad. Wiss. Berlin. 1896. P. 985-1021.

18. Медных $A$. Д. Определение числа неэквивалентных накрытий над компактной римановой поверхностью // Докл. АН СССР. 1978. Т. 239. № 2. С. 269-271.

19. Курош А. Г. Теория групп. М.: Наука, 1967.

20. Зейферт Г., Трельфалль В. Топология. М.-Л.: ГОНТИ, 1938.

21. McCord M. C. Inverse limit sequences with covering maps // Trans. Amer. Math. Soc. 1965. V. 114. P. 197-209.

22. Schori R. M. Inverse limits and homogeneity // Trans. Amer. Math. Soc. 1966. V. 124. P. 533-539.

23. Rogers J. T., Jr., Tollefson J. L. Involutions on solenoidal spaces // Fund. Math. 1971. V. 73. P. 11-19.

24. Ezell C. L. Branch point structure of covering maps onto nonorientable surfaces // Trans. Amer. Math. Soc. 1978. V. 243. P. 123-133.

25. Berstein I., Edmonds A. L. On the construction of branched coverings of low-dimensional manifolds // Trans. Amer. Math. Soc. 1979. V. 247. № 1. P. 87-124.

26. Edmonds A.L., Kulkarni R.S., Stong R. E. Realizability of branched coverings of surfaces // Trans. Amer. Math. Soc. 1984. V. 282. № 2. P. 773-790.

27. Bogatyi S., Gonçalves D. L., Zieschang H. The minimal number of roots of surface mappings and quadratic equations in free groups // Math. Z. 2001. V. 236. № 3. P. 419-452.

28. Kneser $H$. Die Kleinste Bedeckungszahl innerhalb einer Klasse von Flachenabbildungen // Math. Ann. 1930. V. 103. P. 347-358.

29. Hopf H. Zur Topologie der Abbildungen von Mannigfaltigkeiten. I // Math. Ann. 1928. V. 100. P. 579-608.

30. Hopf H. Zur Topologie der Abbildungen von Mannigfaltigkeiten. II // Math. Ann. 1929/1930. V. 102. P. 562-623.

31. Hopf $H$. Über den Defekt stetiger Abbildungen von Mannigfaltigkeiten // Rend. Mat. Appl. (5). 1963. V. 21. P. 275-285.

32. Shepardson C. B. Generalized Hurwitz-Riemann formulas // Indiana Univ. Math. J. 1973. V. 23. P. 277-285.

33. Gabai D., Kazez W. H. The classification of maps of surfaces // Invent. Math. 1987. V. 90. P. 219-242.

34. Натанзон C. M. Топология двумерных накрытий и мероморфные функции на вещественных и комплексных алгебраических кривых // Труды сем. по вектор. и тензор. анализу. 1988. T. 23. С. $79-103$.

35. Натанзон C. M. Топология двумерных накрытий и мероморфные функции на вещественных и комплексных алгебраических кривых. II // Труды сем. по вектор. и тензор. анализу. 1991. Т. 24. С. 104-132.

36. Протопопов A.H. Гомеоморфизмы разветвленных накрытий двумерной сферы // Докл. АН СССР. 1986. Т. 290. № 4. С. 792-795.

37. Khovanskii A. G., Zdravkovska S. Branched covers of $S^{2}$ and braid groups // J. Knot Theory Ramifications. 1996. V. 5. № 1. P. 55-75.

38. Dolcher $M$. Exceptions to $n$-covering for continuous mappings of a plane region // Proc. Internat. Cong. Math. Amsterdam. 1954. V. II. Amsterdam: North-Holland, 1954. P. 95.

39. Isaacs $R$. Iterates of fractional order // Canad. J. Math. 1950. V. 2. P. 409-416.

40. Lojasiewicz $S$. Solution générale de l'équation fonctionnelle $f(f(\ldots f(x) \ldots))=g(x) / /$ Ann. Soc. Polon. Math. 1952. V. 24. P. 88-91. 
41. Rice R. E., Schweizer B., Sklar A. When is $f(f(z))=a z^{2}+b z+c$ ? // Amer. Math. Monthly. 1980. V. 87. P. 252-263.

42. Baker I. N. Fixpoints and iterates of entire functions // Math. Z. 1959. V. 71. P. 146-153.

43. Трохимчук Ю. Ю. О непрерьвных отображениях областей евклидова пространства // Укр. матем. журн. 1964. Т. 16. № 2. С. 196-211.

44. Чернавский A.B. Конечнократные открытые отображения многообразий // Матем. сб. 1964. Т. 65. № 3. С. 357-369.

45. Чернавский $A$. В. Дополнение к статье "О конечнократных открытых отображениях многообразий” // Матем. сб. 1965. Т. 66. №3. С. 471-472.

46. Church V.T., Hemmingsen E. Light open maps on $n$-manifolds // Duke Math. J. 1960. V. 27. №4. P. 527-536.

47. Church V. T., Hemmingsen E. Light open maps on $n$-manifolds. II // Duke Math. J. 1962. V. 28. № 4. P. 607-623.

48. Church V.T., Hemmingsen E. Light open maps on $n$-manifolds. III // Duke Math. J. 1963. V. 30. №3. P. 379-380.

49. Church V. T. Differentiable open maps on manifolds // Trans. Amer. Math. Soc. 1963. V. 109. № 1. P. 87-100.

50. Hemmingsen E. Open simplicial mappings of manifolds on manifolds // Duke Math. J. 1965. V. 32. № 2. P. 325-331.

51. Väisälä J. Discrete open mappings on manifolds // Ann. Acad. Sci. Fenn. Ser. A I Math. 1966. V. 392. P. 1-10.

52. Honkapohja M. Degree and point inverses of mappings on generalized manifolds // Ann. Acad. Sci. Fenn. Ser. A I Math. Dissertationes. 1975. V. 4. P. 1-19.

53. Church VT. Discretemaps on manifolds // Michigan Math. J. 1978. V. 25. № 3. P. 351-357.

54. Church V. T., Timourian J. G. Deficient points of maps on manifolds // Michigan Math. J. 1980. V. 27. № 3. P. 321-338.

55. Ritt J. F. Prime and composite polynomials // Trans. Amer. Math. Soc. 1922. V. 23. № 1 . P. 51-66.

56. Müller P. Primitive monodromy groups for polynomials // Recent developments in the inverse Galois problem. A joint summer research conference, July 17-23, 1993, University of Washington, Seattle, WA, USA / ed. M.D. Fried et al. Providence, RI: Amer. Math. Soc., 1995. P. 385-401. (Contemp. Math. V. 186.)

57. Dorey F., Whaples G. Prime and composite polynomials // J. Algebra. 1974. V. 28. P. 88-101.

58. Fried M. On a theorem of Ritt and related Diophantine problems // J. Reine Angew. Math. 1973. V. 264. P. 40-55.

59. Engstrom H. T. Polynomial substitutions // Amer. J. Math. 1941. V. 63. P. 249-255.

60. Levi $H$. Composite polynomials with coefficients in an arbitrary field of characteristic zero // Amer. J. Math. 1942. V. 64. P. 389-400.

61. Dorfer G., Woracek H. Formal power series and some theorems of J.F. Ritt in arbitrary characteristic // Monatsh. Math. 1999. V. 127. P. 277-293.

62. Schinzel A. Polinomials with special regard to reducibility. Cambridge: Cambridge Univ. Press, 2000.

Московский государственнњй университет дизайна и технологий; Московский государственньй университет им. М.В. Ломоносова; Ruhr-Universität Bochum

E-mail: bogatyi@mech.math.msu.su;

Marlene.Schwarz@ruhr-uni-bochum.de
Поступила в редакцию

14.02 .2001 